\title{
Aluminium Matrix Composites (AA6061/CaSiO3) Obtained by Powder Metallurgy
}

D. Campos Valenzuela ${ }^{1}$, O. Hernández Negrete ${ }^{1}$,*, M.A. Encinas Romero ${ }^{1}$, G. Tiburcio Munive ${ }^{1}$, A. Valenzuela Soto ${ }^{1}$, J.H. Coronado López ${ }^{1}$, F. Brown Bojórquez ${ }^{2}$, H. Esparza Ponce ${ }^{4}$, J. Hernández Paredes ${ }^{3}$

${ }^{1}$ Departamento de Ingeniería Química y Metalurgia, Universidad de Sonora (UNISON), Hermosillo, Sonora, México, ${ }^{2}$ Departamento de Investigación en Polímeros y Materiales, Universidad de Sonora (UNISON), Hermosillo, Sonora, México, ${ }^{3}$ Departamento de Física, Universidad de Sonora (UNISON), Hermosillo, Sonora, México, ${ }^{4}$ Centro De Investigación en Materiales Avanzados (CIMAV), Chihuahua, Chihuahua, México.

* Corresponding author: ofelia.hernandez@unison.mx

Aluminum matrix composite materials (AMCs) are widely used in components for the aerospace and automotive industries [1]. These compounds show a high strength/weight ratio, good mechanical properties and greater durability due to the addition of particles as reinforcements [1,2]. The present work will focus on the study of the reinforcement of AA 6061 alloy with particles of $\mathrm{CaSiO}_{3}$ (wollastonite). Powder metallurgy is widely used to control the dispersion of particles in AMCs [3,4]. The present work aims to study the influence of different contents of $\mathrm{CaSiO}_{3}$ on the microstructure of the alloy matrix and the effect of the powder metallurgy technique by optical microscopy (OM), scanning electron microscopy and energy dispersive X-ray spectroscopy (SEM / EDS).

The preparation of the AMCs was performed by conventional powder metallurgy. AA 6061 aluminum alloy particles obtained by friction were used (figure 1). The $\mathrm{CaSiO}_{3}$ contents used as reinforcement were 0.5, 1.0 and 1.5. wt \% respectively. AA 6061 powders, wollastonite and zinc stearate, were screened using an 80 mesh, after mixing in a vortex set for $15 \mathrm{~min}$. Subsequently, uniaxial pressing was used to obtain compacts of AMCs using 3.4 tons for 5 minutes. Then, the (green) compacts were sintered in $\mathrm{N}_{2}$ atmosphere, heating $10^{\circ} \mathrm{C} / \mathrm{min}$ to $580^{\circ} \mathrm{C}$ for 40 minutes and cooled down in the oven. A metallographic preparation was made consisting of roughing and polishing to study its microstructure.

Figure 1 shows, the mixed powders after uniaxial pressing (greens) and the same samples after sintering. The SEM images (figure 2) show the typical microstructures of the sintered AMCs at different compositions of $\mathrm{CaSiO}_{3}$. All the samples depicted some porosity and according to BSE images and EDS analyses the dark grey areas at grain boundaries in figure 3 a corresponded to the composition of $\mathrm{Al}_{2} \mathrm{O}_{3}$ with $\mathrm{CaSiO}_{3}$, bright contrast particles were found to be Fe-rich oxide as contaminant from the friction tool used to obtain AA6061 powders. 

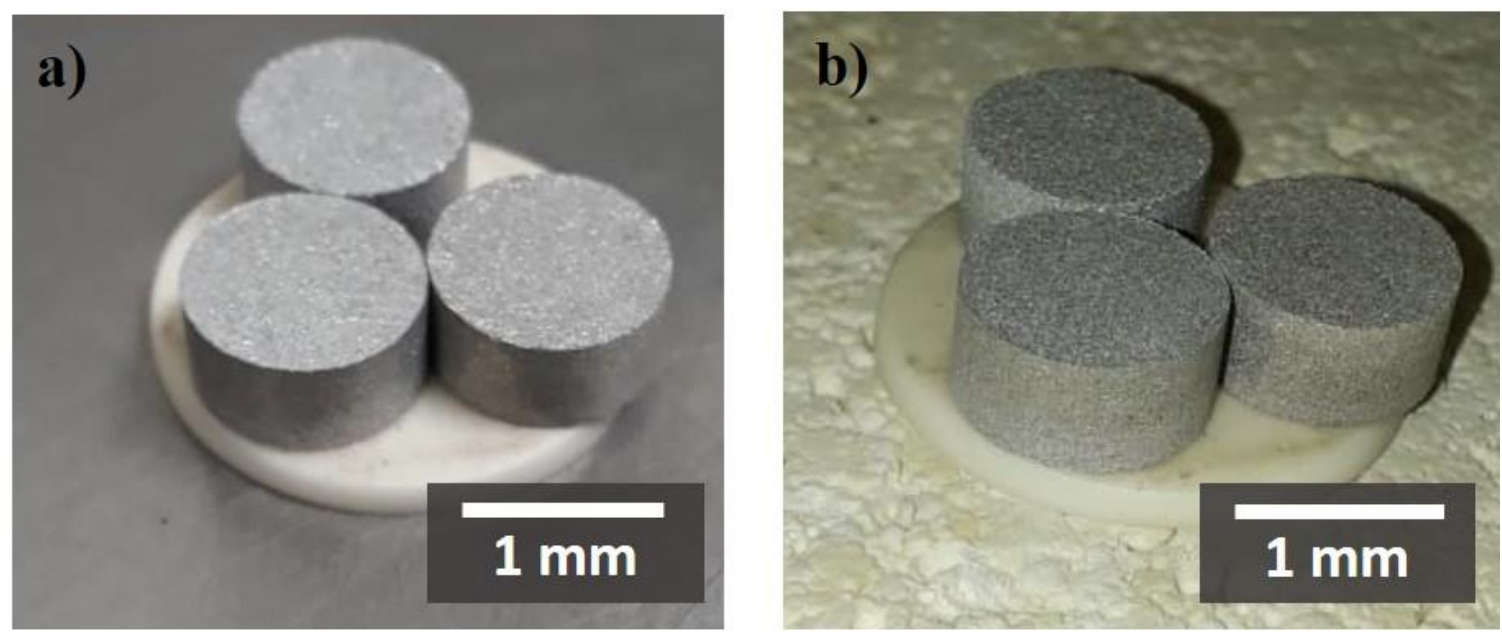

Figure 1. Aluminium Matrix Composites $6061 / \mathrm{CaSiO} 3$, a) Green compacts after uniaxial pressing b) Sintered compacts a $580{ }^{\circ} \mathrm{C}$ for $40 \mathrm{~min}$ in N2 atmosphere.
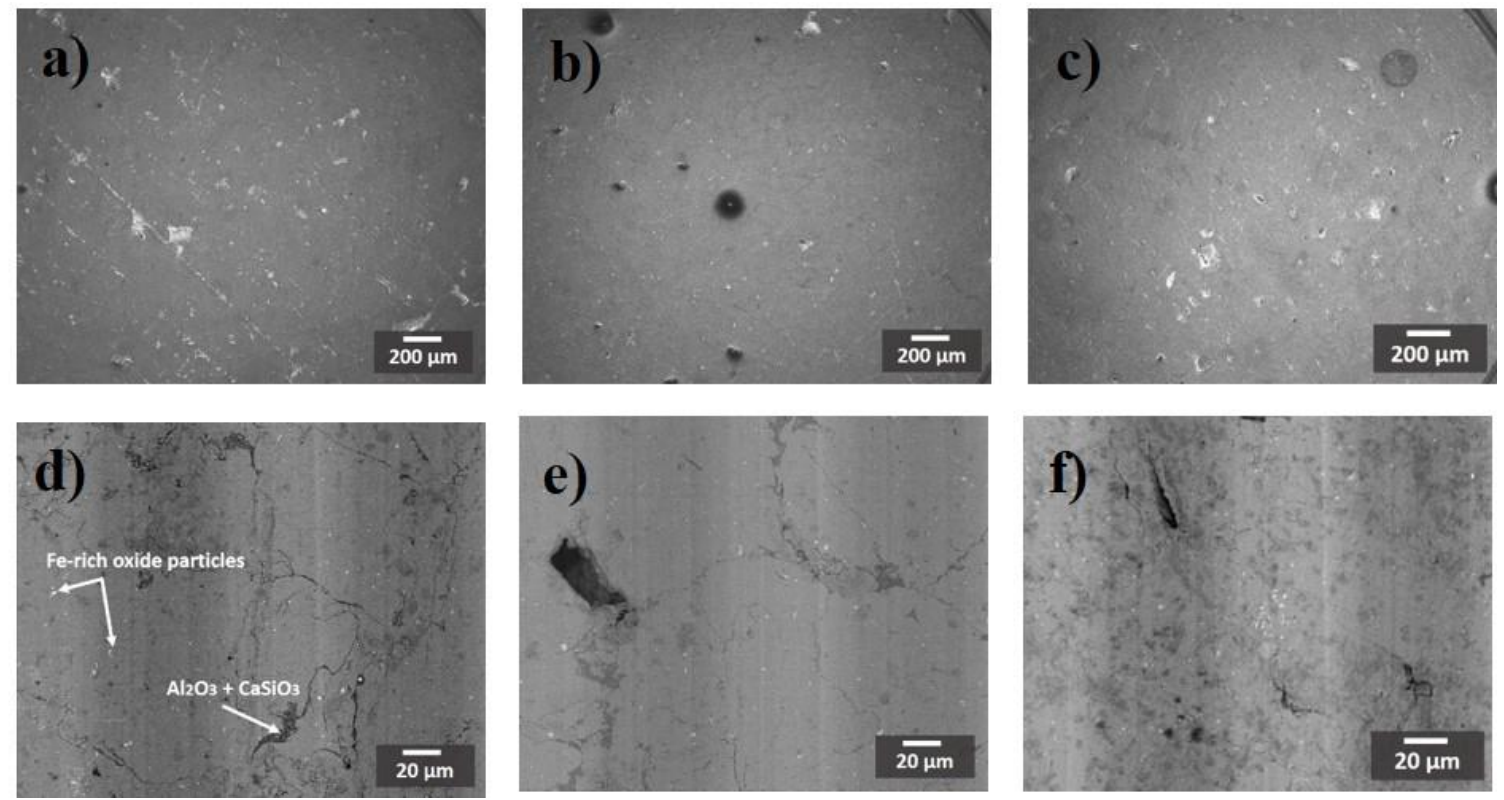

Figure 2. SEC images Aluminium Matrix Composites 6061/CaSiO3, a) $0.5 \%$, b) $1.0 \%$ and c) $1.5 \%$ $\mathrm{CaSiO} 3$ respectively. BSE images of the Aluminium Matrix Composites 6061/CaSiO3, d) $0.5 \%$, e) $1.0 \%$ and f) $1.5 \% \mathrm{CaSiO} 3$ respectively.

\section{References}

[1] C. Yan, W. Lifeng, R. Jianyue Chinese Journal of Aeronautics, Vol. 21, (2008) p.578

[2] E. Madej, Archives of Metallurgy and Materials, Vol. 58. (2013) p.1

[3] S.V.S. Narayana, B. Nageswara and B.P. Kashyap, Composites science and technology 63 (2003) p. 119.

[4] A.K. Surappa. Sadhana 28 (2003) p. 319 